\title{
A Quantum Dot Based Vertical Cavity Optical Modulator for Chip-to-chip Interconnects in Si VLSI
}

\author{
Bin Shi and Ya-Hong Xie \\ Department of Materials Science \& Engineering \\ University of California, Los Angeles, CA 90095-1595 \\ (310) 825-2971 \\ yhx@ucla.edu
}

Chip-to-chip interconnect is becoming the bottleneck for the system performance of current and future generation Si integrated circuits. Optical interconnects are proposed to be the solution, which can overcome the limitations of the conventional interconnection technology ${ }^{1-3}$ In this respect, vertically coupled optical modulator arrays have many advantages over the alternatives of employing actual light emitters such as lasers, on $\mathrm{Si}$. The advantages include simple driver circuits, less added power load on the chip, fewer materials challenges, and other integration issues. III-V quantum-well modulators based on quantum-confined Stark effect have so far been the devices most extensively used in demonstrating dense optical interconnects between chips. But the technology of integration of III-V devices with silicon is always difficult and costly. ${ }^{3}$ In this report, we propose a new optical modulators based on electrical field induced saturation absorption of CdSe quantum dots (QDs). It can be easily integrated with silicon VLSI and can be fabricated on the finished silicon VLSI wafers. In other words, the fabrication of the modulator arrays demands minimum intrusion to the conventional Si CMOS process flow.

The structure of the EA modulator is shown in Fig.1. To the CMOS circuit, it is a simple capacitor. An electroabsorption layer of CdSe quantum dots dispersed in $\mathrm{SiO}_{2}$ is sandwiched in a dielectric vertical cavity composed of two quarter-wave stacks of 4-period $\mathrm{TiO}_{2} / \mathrm{SiO}_{2}$. An ITO layer is used as the top electrode, and the entire device is fabricated by sputter deposition on top of the Al contact pad of the Si CMOS circuit. The CdSe QDs in $\mathrm{SiO}_{2}$ layer is deposited by standard spin-on-glass process.

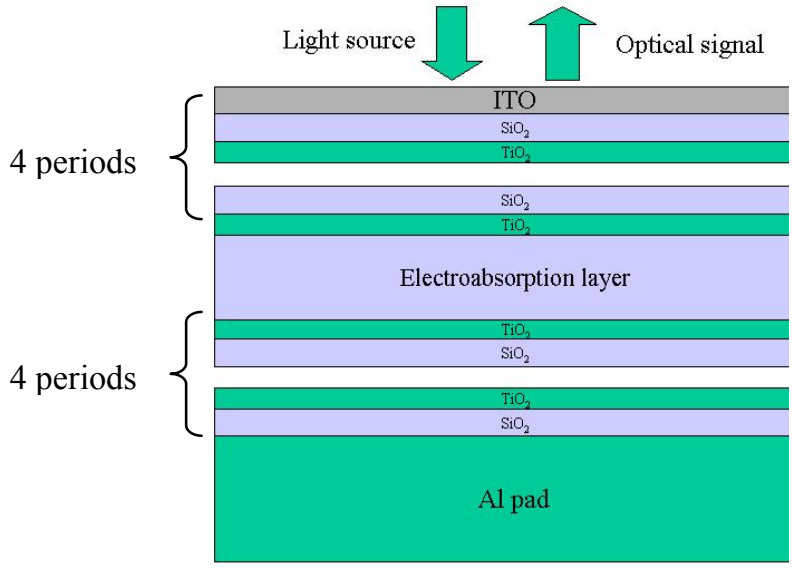

Fig. 1 Schematic view of the modulator structure.

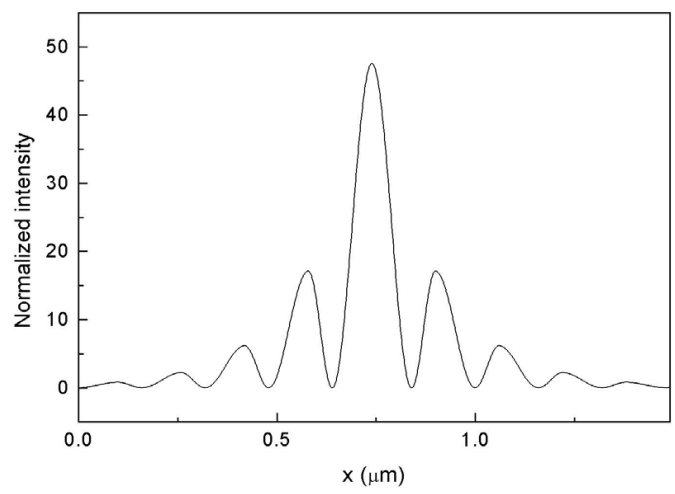

Fig. 2 Light intensity distribution in the modulator.

The principle of electric field controlled absorption in this CdSe QDs embedded in $\mathrm{SiO}_{2}$ matrices system is fundamentally different from the well-known quantum confined Stark effect. It is well known that when the incident light intensity is near the saturation intensity of the quantum dots, the absorption coefficient will decrease with 
increasing excitation intensity. The saturation intensity is directly related to the exciton population relaxation time of CdSe QDs. When a static electric field is applied to the device, the overlap between electron and hole wavefunctions in the quantum dots is altered, resulting in a change in the recombination rate, the exciton population relaxation time, and consequently the saturation intensity. A reduction in the saturation intensity means less light being absorbed by the QD layer at a given incident light intensity. To the optical modulator, this means an increase in the reflectivity of the device.

To operate the device near the absorption saturation limit requires a relatively high incident light intensity $\left(\sim 10^{5} \mathrm{~W} / \mathrm{cm}^{2}\right)$, more than one order of magnitude above that of a typical semiconductor laser, e.g. those used in commercial CD players. The vertical cavity is used to modify the spatial distribution of the incident light intensity from an off-chip CD laser of $635 \mathrm{~nm}$ wavelength. It introduces a resonant state in the photonic band gap of much higher density of states than that in the free space. This function of the vertical cavity can be calculated as shown in Fig.2. Light intensity enhancement by about two orders of magnitude at the CdSe QDs layers is expected. The degree of intensity enhancement can be tailored through the quality factor of the cavity and can easily be increased by another 2 orders of magnitude by adding 3 additional period to each of the mirrors. This will, however, come with an associated narrowing in the bandwidth. Optimization in design will have to be done for a given application.

We use the transfer matrix method to calculate the reflection spectra, and the finite-difference time-domain method to simulate the optical time response of such a device. The results are show in Fig. 3. Modulation depth of deeper than $90 \%$ can be achieved with an insertion loss of lower than $7 \mathrm{~dB}$. The time it takes for the build up of the optical field inside such a device is less than 1 picosecond. This means that below $1 \mathrm{THz}$ operation frequency, the main time delay is due to the electrical charging of the capacitor. For a $5 \mu \mathrm{m} \times 5 \mu \mathrm{m}$ device, $0.1 \mathrm{~mA}$ driving current is required to operate the modulator at $25 \mathrm{GHz}$.
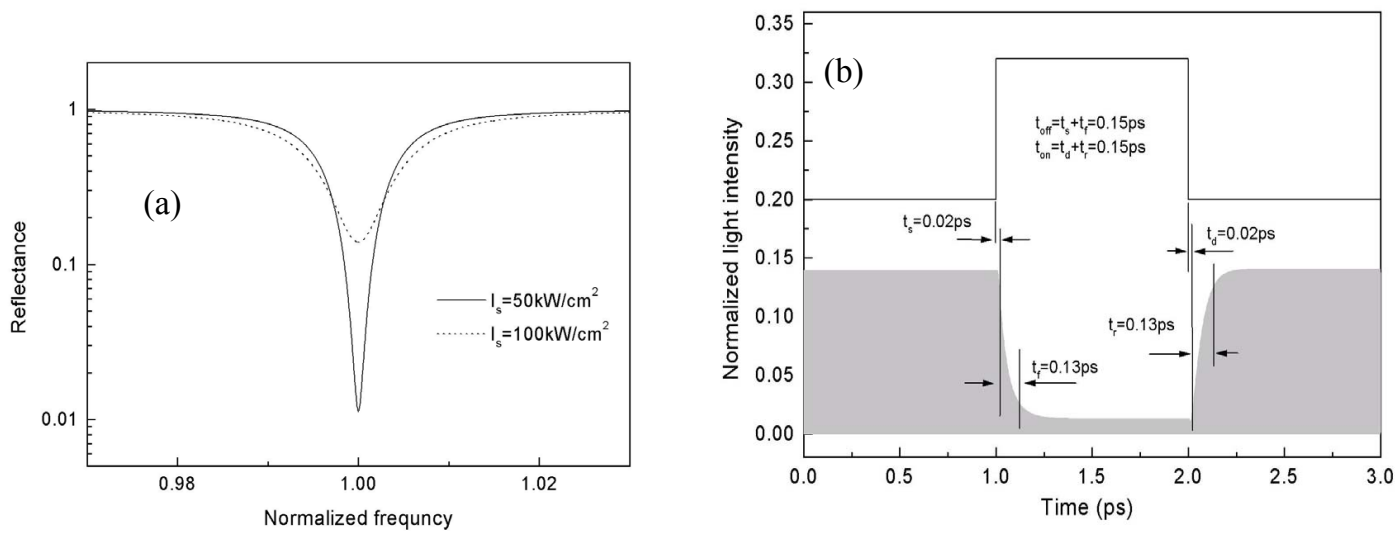

Fig. 3 (a) reflection spectra of the modulator at different saturation intensity, (b) optical time response of such a modulator

The prototype device of the EA modulator was fabricated by radio-frequency (RF) sputtering of $\mathrm{SiO}_{2} / \mathrm{TiO}_{2}$ multilayers and spin-coating of CdSe QDs with Methyl Silsesquioxane by spin-on-glass method. The measured reflection spectra of the device under different biases are shown in Fig. 4. By applying a 28V bias voltage, a modulation depth of $36 \%$ ( $2 \mathrm{~dB}$ contrast ratio) can be achieved with insertion loss of $6 \mathrm{~dB}$. The operation spectral bandwidth of such device is $21 \mathrm{~nm}$. Such modulation performance is comparable with other kinds of silicon-based modulation devices but with great advantages of high frequency operation and ability to make 2-D modulation array. 
Despite the high operating voltage necessary for reasonable modulation depth, this result demonstrates for the first time the feasibility of this novel optical modulator. This worse than predicted performance is due to the low incident light intensity $\left(\sim 1 \mathrm{~W} / \mathrm{cm}^{2}\right.$ from a tungsten halogen lamp) by about 3 orders of magnitude comparing to that from a CD laser used in the measurement. To confirm this, the incident intensity dependence measurement of the modulation depth versus bias voltage was carried out (Fig.5). The proper operation of the modulator requires the laser wavelength to be shorter than the absorption edge of the quantum dots and to coincide with the wavelength of the cavity mode. Furthermore, the cavity mode is determined by the stop band of the quarterwave stacks together with the thickness of the cavity, i.e. the CdSe quantum dot containing $\mathrm{SiO}_{2}$ layer. For the preliminary result shown in fig. 4, the thickness of the dielectric layers were off from the targeted value, leading to the cavity mode being around $520 \mathrm{~nm}$. In the absence of a laser operating at this wavelength, a tungsten halogen lamp instead of a laser was used for the measurement, and thus the high modulation voltage. Further work is underway to correct this problem.

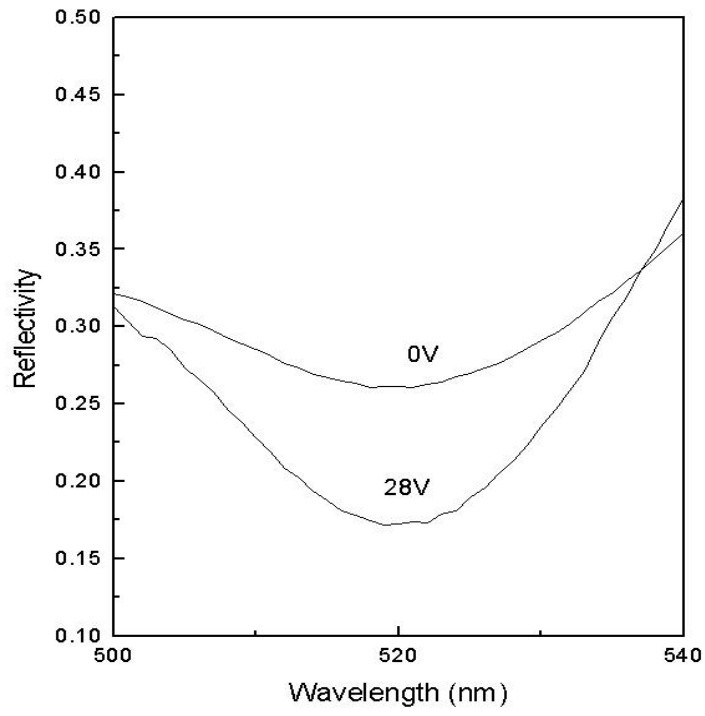

Fig. 4 Spectral dependence of normalized reflectivity at biases of 0 and $28 \mathrm{~V}$

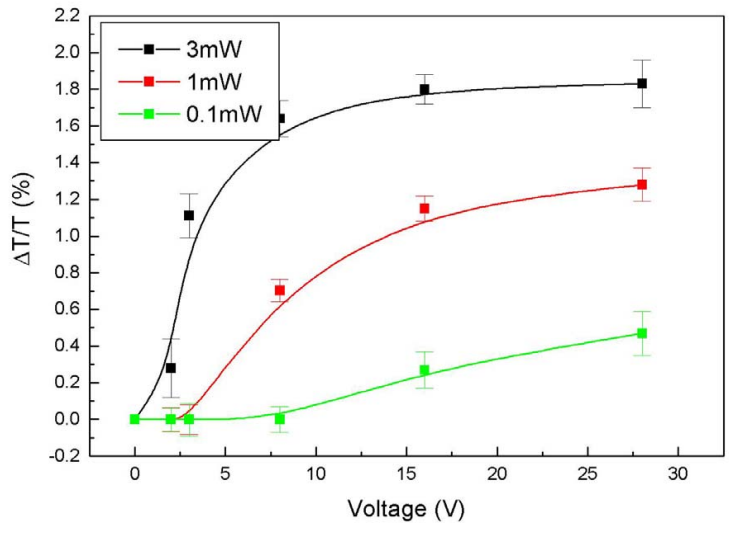

Fig. 5 Field dependent absorption saturation of CdSe quantum dots in solution as a function of the incident light intensity.

In summary, a novel optical modulator is proposed by using a vertical cavity structure in combination with quantum dots materials. It works as a surface reflecting modulator and dense optical interconnects can be obtained by forming 2-dimensional arrays of the modulators. By choosing visible light, Si diode can function as the photodetector. Optical transciever therefore consists of the optical modulator and Si photodetector. Such an optical transceiver can be easily integrated with Si CMOS process because it can be fabricated at the end of the entire process flow.

References:

1. D. A. B. Miller, Proc. IEEE, 88,728 (2000).

2. D. Fey, W. Erhard, M. Gruber, J. Jahns, H. Bartelt, G. Grimm, L. Hoppe, and S. Sinzinger, Proc. IEEE, 88, 838 (2000).

3. D. A. B. Miller, IEEE J. Select. Topics Quantum Electron., 6, 1312 (2000). 